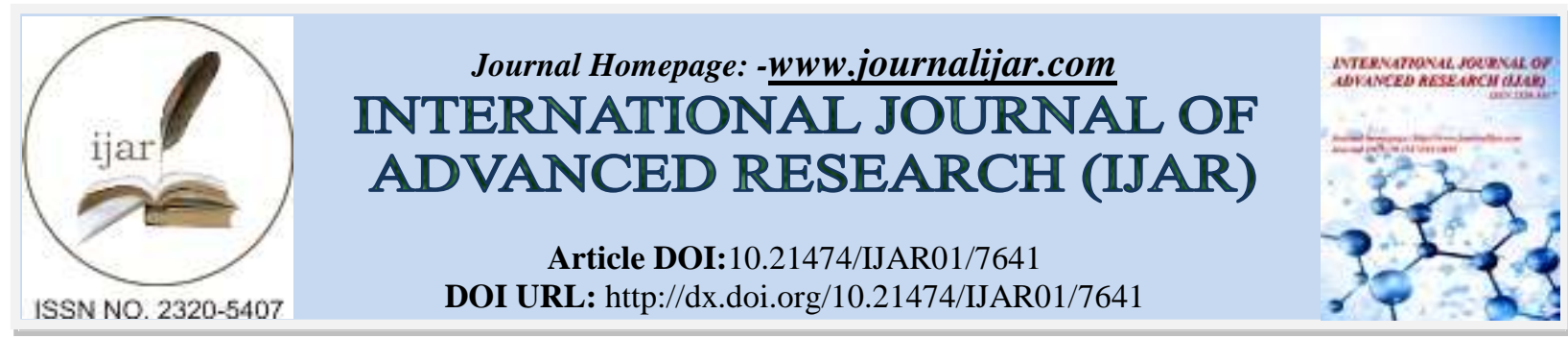

RESEARCH ARTICLE

\title{
EFFICACY OF THORACIC PARAVERTEBRAL BLOCK ANESTHESIA IN BREAST AUGMENTATION SURGERY.
}

\section{Sarh Alaeed ${ }^{1}$, Rahaf Mandura ${ }^{2}$, Maan Kattan ${ }^{3}$, Basim Alsaywid ${ }^{4}$ and Wesam Abuznadah ${ }^{5}$.}

1. General physician, King Salman Armed Forces Hospital, Tabuk, Saudi Arabia.

2. Ophthalmology teaching assistant, King Abdulaziz university, PGY 2 Saudi Board of Ophthalmology, Jeddah, Saudi Arabia.

3. Anesthesia department chairman, Consultant anesthesiologist and pain physician, King Abdulaziz Medical City, Jeddah, Saudi Arabia.

4. Pediatric Urologist and Epidemiologist, King Saud Bin Abdulaziz University for Health Sciences, King Abdulaziz Medical City, Jeddah, Saudi Arabia.

5. Assistant professor of vascular Surgery, College of Medicine, King Saud bin Abdulaziz University for Health Sciences, King Abdulaziz Medical City, Jeddah, Saudi Arabia.

\section{Manuscript Info}

(.........................

Manuscript History

Received: 02 July 2018

Final Accepted: 04 August 2018

Published: September 2018

\section{Keywords:-}

breast augmentation, paravertebral block, bupivacaine, post-op analgesia, regional, surgery

\section{Abstract}

Elective cosmetic Breast augmentation is a highly accomplished operation in Kingdom of Saudi Arabia, it can be combined with prominent post-operative pain being a source of concern for patients and their healthcare providers. General anesthesia (GA) has been a standard technique for this surgery.

Methodology: A retrospective chart review of 131 patients was conducted at king Abdul Aziz Medical City, Jeddah in the period of 2002-2012. Our study sample was 60 patients divided into two groups, one who underwent GA alone, and the other one underwent GA with bilateral single injection at single level T3-T4 TPVB of $0.2-0.5 \%$ bupivacaine + epinephrine $5 \mathrm{mcg} / \mathrm{ml} \mathrm{(1:200000)}$ before the induction of GA. The primary outcome assessed was the amount of post-operative analgesic use in both groups in the $1^{\text {st }} 24$ hours.

Results: Overall, the patients' traits and anesthetic technique were proportionate between the two groups. Bilateral single injection TPVB significantly decrease the need for analgesics $(=10.549,1 \mathrm{df}, \mathrm{P}=0.001)$, and antiemetic needs $(=16.98,1 \mathrm{df}, \mathrm{P}=0)$. There was a statistically difference in the duration of hospital stay between the 2 groups $(=4.23$, $1 \mathrm{df}, \mathrm{P}=0.04)$. The pain visual analogue score in the group with the TPVB was significantly decreased $(=4.23,1 \mathrm{df}, \mathrm{P}=0.01)$.

Conclusion: we found a statistically significant difference between both groups, which present efficacy of using TPVB. We advocate the use of TPVB in all breast augmentations.

Copy Right, IJAR, 2018,. All rights reserved.

\section{Introduction:-}

Although elective cosmetic breast augmentation is a highly accomplished operation in Saudi Arabia, it can be combined with prominent post-operative pain being a source of concern for patients and their healthcare providers. 
The most prevailing operated type of breast augmentation is the sub muscular type because it has a low rate of complications and capsular contracture. In this type of augmentation, the pectoralis major muscle will be crosscut along the medial and inferior borders allowing the implant to rest underneath the breast mound, which is the main factor causing the marked pain postoperatively. ${ }^{(1)}$ Moreover, approximately all patients who went through this operation are healthy entities, which will make them more reliable to be stressed by the postoperative pain. Application of all these aspects leads to an increased prescription of analgesics in this type of surgery. General anesthesia (GA) has been a standard technique for breast augmentation surgery. ${ }^{(2)}$ In the last six years, regional anesthesia using thoracic paravertebral block was introduced to King Abdulaziz Medical City, Jeddah, (KAAMC) with the target of lessening the discomfort in the post-operative period. The preference of thoracic paravertebral block over general anesthesia is the arrangement of effective surgical anesthesia along with prolonged postoperative analgesia. ${ }^{(3)}$

The paravertebral block was first done in Germany by Hugo Sellheim in 1905. His goal was to replace the spinal anesthesiatechnique, free from its feared risks of heart and lung related deterioration. A resident of General surgery in Germany, Arthur Lawen, formed a special study of this new procedure. Through the painstaking injection of procaine at the origin of each spinal nerve in plentiful of patients with abdominal pain who went through laparotomy, it was valuable in calming the abdominal muscle spasm in patients with an acute abdomen. ${ }^{(4)}$ The thoracic paravertebral block (TPVB) performance for postoperative analgesia in patients undergoing thoracic or abdominal surgical operations is well founded. ${ }^{(5)}$ Reports regarding the effects of this intervention on anticipated pain in breast augmentation has been recorded but not quantitatively examined in the matter of narcotic use. ${ }^{(6)}$ The thoracic paravertebral space is a wedge-shaped space lying on two sides of the vertebral column. The vertebral body, intervertebral disc, intervertebral foramina and its contents forming its base. The anterolateral border is formed by the parietal pleura and posterior border by a costotransverse ligament. The apex is continuous with intercostal space laterally and with sub pleural space at the tip of transverse process. ${ }^{(7)}$

The thoracic paravertebral block is a procedure of a single shot of local anesthetic which injected bilaterally adjoining to the thoracic vertebrae close to where the spinal nerves rise from the intervertebral foramina. This result in blockage of somatic and sympathetic nerve in several thoracic dermatomes below and above the site of injection. (8)

Our clinical impression from patient medical records review was that the use of bilateral thoracic paravertebral block by a single injection of local anesthetic $0.2-0.5 \mathrm{ml} / \mathrm{kg}$ of bupivacaine given at a single site from T3 to T4 has a discernible role in controlling post-operative pain and thereafter analgesic consumption.

The purpose of this study is to assess post-operative analgesic use in the first twenty-four hours in patients who went through thoracic paravertebral block and general anesthesia and its impact on antiemetic medication use, recovery room stays, a total duration of hospitalization and the possible hazards of the thoracic paravertebral block.

\section{Objective:-}

This study was attempted to quantitatively report the capability of thoracic paravertebral block in rolling the pain which develop postoperatively in primary breast augmentation surgery and its consequence on analgesic and antiemetic use within the first 24 hours postoperatively, recovery room stay, a total duration of hospitalization and complications.

\section{Methods and Materials:-}

After getting the acceptance of the Hospital Ethics Committee, a retrospective chart review of 131 patients whowent through breast augmentation mammoplasty was conducted at KAAMC in the period of 2002-2012. All patients aged 18-55 years, American Society of Anesthesia (ASA) physical status class 1 and 2 were included. We excluded 53 patients who were admitted for 2ry implant exchange, ASA physical status class 3 and above, patients on chronic pain medication and those with any other major surgery performed at the same time. Our study sample was 60 patients divided into two groups A and B. Group A involved 32 patients who went through breast augmentation under general anesthesia alone, and group B included 28 patients who went through breast augmentation under general anesthesia with bilateral single injections at single level T3-T4 thoracic paravertebral block of $0.2-0.5 \%$ bupivacaine + epinephrine $5 \mathrm{mcg} / \mathrm{ml}(1: 200000)$ before the induction of general anesthesia. 
Measurements:-

1. Postoperative analgesic and antiemetic consumption during the first 24 hours.

2. The time to first analgesic demand.

3. Visual Analogue Scale (VAS) during recovery room stay.

4. Duration of recovery room stay.

5. Total duration of hospitalization.

6. Possible complications including intraoperative hypotension ( $\mathrm{SBP}<80)$, oxygen desaturation $(\mathrm{SaO} 2<87)$, estimated blood loss.

\section{Statistical analysis:-}

Simple descriptive statistics was created for patients' demographics including mean and standard deviation for continuous variables and proportion for categorical data. in order to compare between proportions, we used two sided chi-square, and a $\mathrm{P}$ value $<0.05$ was considered significant. To compare between means, independent sample T-test was calculated.

\section{Results:-}

The patients' traits and anesthetic procedure were proportionate between the two groups of patients. There was no significant variation between them with reference to demographic data, body mass index and ASA physical status $(\mathrm{P}=0.9,0.83$ and 0.64 respectively).

The diversity in the need for analgesia in the first 24 hours postoperatively was highly significant. It was markedly decreased in group B who received TPVB. $72.7 \%$ of group B patients did not demand any analgesia for 24 hours after the surgery as compared with $27.3 \%$ of group A patients $(=10.549,1 \mathrm{df}, \mathrm{P}=0.001)$. Also, the need for antiemetic in this group was significantly less $(=16.98,1 \mathrm{df}, \mathrm{P}=0)$.

Among group B who demanded analgesia, there was a trend toward lesser consumption of morphine and pethidine in milligrams postoperatively compared to group A during the first 24 hours, however, it did not reach the level of statistical significance $(\mathrm{t}=1.08,33 \mathrm{df}, \mathrm{P}=0.28)(\mathrm{t}=1.32,12 \mathrm{df}, \mathrm{P}=0.21)$ respectively. A mean of $8.5 \pm 6.4 \mathrm{mg}$ morphine was consumed by patients of group B compared to $13.4 \pm 17.14 \mathrm{mg}$ by patients of group A and the mean of pethidine consumption was $50 \pm 0 \mathrm{mg}$ in group B compared to $1532 \pm 74.6 \mathrm{mg}$ in group A.

Similarly, among group B patients who demanded antiemetic, a trend toward lesser consumption of metoclopramide antiemetic was found, a mean of $10 \pm 0 \mathrm{mg}$ in group B compared to $13.21 \pm 8.2 \mathrm{mg}$ in group A $(\mathrm{t}=10.94,18 \mathrm{df}$, $\mathrm{P}=0.071)$.

The Total duration of hospitalization was considerably shorter in group B $(=4.23,1 \mathrm{df}, \mathrm{P}=0.04) .71 .4 \%$ of group B patients were discharged on the operation day in comparison to only $28.6 \%$ of group A patients.

The pain visual analog score in group $\mathrm{B}$ was significantly decreased $(=4.23,1 \mathrm{df}, \mathrm{P}=0.01)$ (table 6$)$. There was a marked variation in the mean of latency to the first analgesic required (4.06 \pm 7.19 hours. vs. $8.26 \pm 7.41$ hours, $\mathrm{P}=0.03$ ). Only $31.6 \%$ of group $\mathrm{B}$ required immediate postoperative rescue analgesia or analgesia administration within 30 minutes of surgery as compared to $68.4 \%$ of group A patients $(=4.82,1 \mathrm{df}, \mathrm{P}=0.028)$.

There was no compelling variation in the length of recovery room stay $(\mathrm{P}=0.48)$. There was an apparent distinction in the magnitude of intraoperative systolic blood pressure (SBP) drop between the two groups. Group B had more drop in SBP compared to group A ( $\mathrm{t}=4.45,58 \mathrm{df}, \mathrm{P}=0.05)$ (table 8). Intraoperative oxygen saturation and the amount of intraoperative blood loss had no statistical significance ( $\mathrm{P}=0.6,0.9$ respectively). The mean time was spent in the induction of both types of anesthesia had no statistically significant difference $(t=2.41,56 \mathrm{df}, \mathrm{P}=0.09)$. the mean time that was spent to perform TPVB+GA in group B patients was $19 \pm 8$ minutes compared to $14 \pm 5$ minutes spent to perform GA alone in group A patients. So, the mean time to perform the block was 5 minutes.

\section{Discussion:-}

The outcome of this study advocate that TPVB in combination with GA provides improved pain control during the first 24 hours after breast augmentation surgery when correlated to GA alone. Patients with bilateral single site TPVB at the level of T3-4, showed statistically significant reduction in the demand for analgesia in the first 24 hours 
postoperatively indicating that TPVB is an efficient opioid saving strategy and consecutively a tool for a reduction in opioid- induced adverse effects in patients receiving TPVB.Significantly lower demand for antiemetic indicative of lower incidence of nausea and vomiting which might develop postoperatively in patients who acquired the block was interpreted by their lower morphine consumption. Various researches demonstrated that the more the dose of morphine consumption in the postoperative period, the higher the tendency to develop nausea and vomiting postoperatively. We concluded that patients going through breast augmentation surgery using TPVB technique were less likely to experience nausea or vomiting in the immediate postoperative interval than patients having GA alone.

Inadequately controlled postoperative pain may defer recovery, lead to extended hospitalization periods, increase medical costs, and it is major burden to surgical patients. Many studies measured the workability of TPVB in favor of the hospitalization periods reduction and to allow patients to be discharged on the day of surgery and ambulate normally after breast surgery. In our study, the results are in agreement with previous studies. It also showed longer time to the first rescue analgesic, lower visual analog score values. Considering the time added for the sake of the anesthesiologist, our results conducted no statistically marked variation in the time spent for induction of both types of anesthesia. Thus we concluded that TPVB is an effective, cost-saving and not time-consuming anesthetic technique.

Clearly, significantly more intraoperative drop in systolic blood pressure was observed in group B with TPVB+GA. Although statistically significant, the magnitude of the difference in intraoperative systolic blood pressure drop between the two groups was small $(82.2 \pm 6.2 \mathrm{mmHg}$ vs. $83.2 \pm 10.8 \mathrm{mmHg})$ and the postoperative benefit that TPVB offers to the patients may justify this minor complication. Moreover, there was no statistical significance for serious complication including intraoperative oxygen desaturation or intraoperative bleeding.

Other complications of TPVB include the probability of insensible injection and spreading into the epidural space. This probability for spreading accentuate the definitive end point lacking at the entrance of the paravertebral space. Lungs' Pleura is extending down to T12 level,one more technical obstacle could be a pneumothorax caused by a deep needle penetration at the level of thorax. ${ }^{(9)}$ in spite of these hazards were not reported in this study, and regarding the safety evaluation of this technique we prompt performing aprospective study over large samples.

\section{Conclusion:-}

This is the first report in Saudi Arabia that proved the efficacy of thoracic paravertebral block in markedly reducing the pain and the analgesic usage within the first 24 hours postoperatively in addition to allocate the patient with the choice of ambulatory discharge. The authors accomplished that its advantages are convincing, and they encourage its application in all breast augmentations because of its cost effectiveness. This study is only the first step toward a wider randomized controlled trial to be done.

\section{References:-}

1. Jabs, D., B.G. Richards, and F.D. Richards, Quantitative effects of tumescent infiltration and bupivacaine injection in decreasing postoperative pain in submuscular breast augmentation. Aesthetic Surgery Journal, 2008. 28(5): p. 528-33.

2. Desai, P.M., Thoracic paravertebral block. British Journal of Anesthesia, 1999. 82(1): p. 149-50.

3. Richardson J, Lonnqvist PA. Thoracic paravertebral block. British Journal of Anesthesia 1998;81:230-238

4. Moussa, A.A., Opioid saving strategy: bilateral single-site thoracic paravertebral block in right lobe donor hepatectomy. Middle East J Anesthesiol, 2008. 19(4): p. 789-801.

5. Siniscalchi A, Begliomoni B, De Pietri L, Et al: Increased prothrombin time and platelet counts in living donor right hepatectomy: implications for epidural anesthesia. Liver Transpl;10:1144-1149, 2004.

6. Ng A, Parker J, Toogood L, Et al: Does the opioid-sparing effect of rectal diclofenac following total abdominal hysterectomy benefit the patient? British Journal of Anesthesia; 88:714-15, 2002.

7. Eason Mj,Wyatt R: Paravertebral thoracic block-a-reappraisal. Anesthesia; 34:638-42, 1979.

8. Wallace MS, Wallace AM, Lee J, Dobke MK. Pain after breast surgery: a survey of 282 women. Pain 1996;66:195-205.

9. Apfelbaum JL, Chen C, Mehta SS, Gan TJ. Postoperative pain experience: results from a national survey suggest postoperative pain continues to be undermanaged. AnesthAnalg2003;97:534-540.

10. Liu N, Kuhlman G, Dalibon N, Moutafis M, Levron JC, Fischler M. A randomized, double-blinded comparison of intrathecal morphine, sufentanil and their combination versus IV morphine patient-controlled analgesia for 
post thoracotomy pain. AnesthAnalg 2001; 92: 31-6 11- Vogt A, Stieger D S, TheurillatCand, Curatolo M, Single-injection thoracic paravertebral block for postoperative pain treatment after thoracoscopicsurgery ,British Journal of Anesthesia 2005;95(6): 816-21 12-E Coveney, C R Weltz, R Greengrass, J D Iglehart, G S Leight, S M Steele, and H K Lyerly, Use of paravertebral block anesthesia in the surgical management of breast cancer: experience in 156 cases, Annals of Surgery Journal 1998 April; 227(4): 496-501.

11. Quinn AC, Brown JH, Wallace PG, Asbury AJ. Studies in postoperative sequelae. Nausea and vomiting--still a problem. Anesthesia. 1994 Jan;49(1):62-65.

12. Richardson J, Sabanathan S. Thoracic paravertebral analgesia. ActaAnaesthesiol Scand. 1995 Nov;39(8):10051015 15-V Bhuvaneswari, Jyotsna Wig, Preethy J Mathew, and Gurpreet Singh, Post-operative pain and analgesic requirements after paravertebral block for mastectomy: A randomized controlled trial of different concentrations of bupivacaine and fentanyl, Indian J Anaesth. 2012 Jan-Feb; 56(1): 34-39 16-Klein SM, Bergh A, Steele SM, Georgiade GS, Greengrass RA. Thoracic paravertebral block for breast surgery, AnesthAnalg. 2000 Jun;90(6):1402-5. 